\title{
Preface to Second Part of Special Issue on Mixtures
}

\author{
Harishankar Ramachandran ${ }^{1}$
}

Published online: 28 July 2017

(C) Indian Institute of Technology Madras 2017

The March issue presented papers on the continuum theory of mixtures, which allows for a systematic study of mixture by taking into account the individual responses of its constituents and their interactions. The aim of this special issue was to bring together researchers working in mixture theory with different thematic foci (experiments, development of mathematical models, numerical simulations in real world applications) and communicate the ideas between the experimentalists, engineers, physicists interested in the development of mathematical models and mathematicians oriented on numerical methods.

The leading article in that issue was not included due to an oversight, and is included here instead. This article presents a brief remark on different aspects of mixture theory. This paper highlights the breadth of recent applications of mixture theory. A brief note on the boundary conditions and their related issues is made. Further, the need and scope for experimental design and measurement techniques for validation of mixture models is presented. Finally, the authors comment on mathematical issues concerning numerical simulations of mixture models.

Harishankar Ramachandran

(Editor in Chief)

Harishankar Ramachandran

hsr@ee.iitm.ac.in

1 Indian Institute of Technlology Madras, Chennai, India 